\title{
Fortalecimento da capacidade institucional para pesquisa em saúde equitativa: lições da América Latina e do Caribe*
}

\author{
Marshall K Tulloch-Reid ${ }^{1}$, Nancy Gore Saravia ${ }^{2,3}$, Rodolfo J Dennis ${ }^{4}$, Andrés Jaramillo,6, Luis Gabriel \\ Cuervo7, Susan P Walker ${ }^{1}$ e Luis Alejandro Salicrup ${ }^{78}$
}

\begin{abstract}
Marshall Tulloch-Reid e colegas afirmam que o desenvolvimento da capacidade de pesquisa na América Latina e no Caribe demanda investimento tanto nos indivíduos quanto nas instituições regionais
\end{abstract}

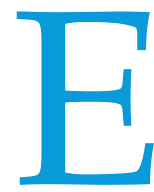
xiste atualmente um maior reconhecimento dos determinantes políticos, econômicos, ambientais e sociais da saúde. A consequência disso foi um aumento da demanda por pesquisa em saúde global, com participação dos países de baixa e média renda, para cumprir os objetivos de desenvolvimento sustentável das Nações Unidas. ${ }^{1}$ Além de financiamento, são necessários recursos humanos e infraestrutura suficientes para que esses países se tornem parceiros em igualdade de condições com instituições de pesquisa internacionalmente reconhecidas em países de alta renda que definem as agendas de pesquisa e cuidam para que o programa global de pesquisa em saúde reflita suas necessidades. A limitada capacidade de pesquisa e as grandes disparidades entre países de alta renda e países de baixa e média renda no tocante à capacidade de

\footnotetext{
* Tradução oficial para o português realizada pela Organização Pan-Americana da Saúde. Em caso de discrepância, prevalecerá a versão original em inglês.

1 Professor, Instituto do Caribe para Pesquisa em Saúde, Universidade das Índias Ocidentais, Kingston, Jamaica. $₫$ marshall.tullochreid@uwimona.edu.jm

2 Diretora, Centro Internacional de Entrenamiento e Investigaciones Médicas, Cali, Colômbia
}

produzir e usar pesquisas para a saúde contribuíram para a falta de evidências para informar a prática e orientar as

políticas de saúde regionais e globais, o que perpetuou as desigualdades em saúde. ${ }^{2}$

\section{MENSAGENS-CHAVE}

- O desenvolvimento sustentável de recursos humanos requer o fortalecimento institucional e a criação de equipes interdisciplinares de pesquisa, e não só o treinamento individual de cientistas.

- O emprego e empoderamento de instituições locais capazes de adaptar o treinamento para satisfazer as necessidades locais e internacionais é crucial para melhorar a pesquisa em países de renda baixa e média em todo o mundo.

- Parcerias justas, respeitosas e estratégicas, associadas a investimentos financeiros em infraestrutura, são necessárias para implementar e manter programas de treinamento sustentáveis em países de baixa e média renda.

- É necessário que organismos de financiamento nacionais e internacionais invistam em recursos humanos e oportunidades de carreira em pesquisa, bem como em infraestrutura física para pesquisa em saúde.

- A avaliação formal do êxito do treinamento em países de baixa e média renda na América Latina e no Caribe é necessária para demonstrar melhor seu efeito sobre a equidade em saúde e pesquisa e para promover maior apoio a esses esforços

\footnotetext{
Diretora, Universidad Icesi, Cali, Colômbia

4 Professor, Departamento de Epidemiología Clínica y Bioestadística, Pontificia Universidad Javeriana, Bogotá, Colômbia

5 Coordenador de projetos e cursos, Centro Internacional de Entrenamiento e Investigaciones Médicas, Cali, Colômbia

6 Coordenador de projetos e cursos, Universidad Icesi, Cali, Colômbia
}

\begin{abstract}
Assessor sênior, pesquisa em sistemas de saúde Serviços de saúde e unidade de acesso, sistemas de Departamento de sistemas e serviços de saúde da Organização Pan-Americana da Saúde/Organização Mundial da Saúde, Washington, D.C., EUA 8 Assessor sênior para pesquisa em saúde global. Centro para a Saúde Global, Instituto Nacional do Câncer, Institutos Nacionais de Saúde dos Estados Unidos, EUA
\end{abstract}


Houve um aumento das pesquisas em alguns países latino-americanos, mas vários territórios do Caribe estão entre aqueles com o menor nível de resultados de pesquisa médica. ${ }^{3}$ Por meio de sua Política de Pesquisa para a Saúde, a Organização Pan-Americana da Saúde (OPAS), escritório regional da Organização Mundial da Saúde (OMS), defende o fortalecimento da pesquisa nacional em todos os Estados Membros para promover a equidade em saúde e o desenvolvimento socioeconômico. ${ }^{2}$

Nós descrevemos os resultados e as lições aprendidas com três estudos de caso, identificados pela OPAS, que discorrem sobre as estratégias adotadas para desenvolver a capacidade de pesquisa na América Latina e no Caribe. $\mathrm{O}$ primeiro estudo descreve a capacitação para pesquisa por colaboração Norte-Sul e é um exemplo da criação de redes latino-americanas. O segundo foi desenvolvido inicialmente por cooperação bilateral, seguida da formação de redes nacionais e internacionais. Tornou-se um modelo para a colaboração Sul-Sul. O terceiro estudo de caso usa várias estratégias para aproveitar a infraestrutura existente. É um exemplo de cooperação técnica entre a OPAS e os países de língua inglesa do Caribe.

\section{COLABORAÇÃO NORTE-SUL: A REDE INTȨRNACIONAL DE EPIDEMIOLOGIA CLÍNICA (INCLEN)}

Na década de 1980, a Fundação Rockefeller patrocinou a criação da INCLEN para aproximar a saúde pública da medicina clínica, com a finalidade de melhorar a saúde nos países em desenvolvimento. ${ }^{4}$ A INCLEN concedeu a jovens do corpo docente clínico de faculdades de medicina no sudeste asiático, na China, na Índia, na África e na América Latina bolsas para cursos de mestrado em epidemiologia clínica na América do Norte e na Austrália. ${ }^{4}$

A Fundação Rockefeller logo constatou a necessidade de centros acadêmicos de apoio em outros países, além dos existentes na América do Norte e na Austrália, para manutenção da iniciativa da INCLEN. Estabeleceu então parcerias com escolas de medicina e saúde pública locais e deu apoio financeiro para a criação de unidades de epidemiologia clínica, incentivando a especialização em bioestatística, economia da saúde e ciências sociais. ${ }^{4,5}$ A fundação também providenciou treinamento em liderança e gestão. ${ }^{4}$ Em um período de 10 anos, criaram-se várias unidades de epidemiologia clínica, que se tornaram centros regionais de excelência para treinamento e pesquisa, com equipes capazes de responder às necessidades regionais de pesquisa. Para reforçar as iniciativas de colaboração regional, criou-se a Rede Latino-Americana de Epidemiologia Clínica (LatinCLEN) em 1991.

A LatinCLEN não teve êxito em todos os lugares, mas atualmente está presente em sete países. As instituições membros oferecem capacitação em epidemiologia clínica, bioestatística e economia da saúde, incluindo um programa de doutorado e nove de mestrado. A LatinCLEN enfrentou as necessidades regionais e realizou pesquisa de alto impacto em saúde mental, saúde materno-infantil, avaliação econômica em saúde, revisões sistemáticas, avaliação de programas e diretrizes clínicas (Quadro 1). Durante os últimos 20 anos, influenciou e liderou a cooperação com as colaborações Cochrane e Campbell. ${ }^{6}$

\section{COLABORAÇÃO SUL-SUL: CIDEIM - CENTRO REGIONAL DE TREINAMENTO NAS AMÉRICAS}

O Centro Internacional de Entrenamiento e Investigaciones Médicas (CIDEIM) na Colômbia é um bom exemplo de transformação de uma parceria bilateral Norte-Sul em um modelo para a colaboração Sul-Sul. O centro surgiu como uma missão de assistência técnica bilateral em 1961, apoiada por uma subvenção dos Institutos Nacionais de Saúde dos EUA para a Universidade de Tulane. ${ }^{7}$ O centro dedicava-se predominantemente à pesquisa em saúde. Firmou parceria com a Faculdade de Medicina da Universidade do Valle e estava localizado em seu campus até 1974. No momento, o COLCIENCIAS, organismo nacional colombiano para promoção e financiamento de ciência e tecnologia, divide a direção com a Universidade de Tulane. $\mathrm{O}$ financiamento multilateral de pesquisas e as subvenções institucionais concedidas por COLCIENCIAS e organismos internacionais, durante a década de 1980, criaram as bases para a transição do CIDEIM a uma instituição de pesquisa biomédica colombiana sem

\section{Quadro 1: Rede Latino-Americana de Epidemiologia Clínica (LatinCLEN)}

A LatinCLEN visa ao fortalecimento de sistemas de saúde nacionais e regionais por meio de pesquisa sobre a efetividade e a eficiência de intervenções de saúde e os determinantes de saúde e doença. Desde sua criação, os centros da LatinCLEN treinaram mais de 400 epidemiologistas clínicos e tiveram uma produção científica de mais de 1.200 publicações. ${ }^{6}$ Os centros da LatinCLEN e seus membros exerceram um papel de liderança na avaliação econômica de intervenções em saúde. Eles aperfeiçoaram métodos para vincular diretrizes clínicas à avaliação econômica em saúde, além de estabelecerem padrões para avaliação de evidências econômicas de diferentes fontes. A rede influenciou as políticas de saúde e de pesquisa, bem como o trabalho de agências de desenvolvimento e organizações intergovernamentais na região.

A pesquisa da LatinCLEN abrange:

- Desenvolvimento e validação de modelos econômicos para avaliar a carga de doença atribuível ao tabagismo e o custoefetividade de intervenções de controle do tabagismo na Argentina e em outros seis países em 2011 (Grupo de Pesquisa sobre Tabaco da LatinCLEN)

- Avaliação econômica de programas de reabilitação cardiovascular com exercício físico para pacientes com insuficiência cardíaca crônica na Colômbia em 2016

- Determinação do impacto do Programa Mãe-Canguru na Colômbia

- Avaliação do custo-efetividade de novos fármacos para pacientes com fibrilação atrial e síndrome coronariana aguda no Chile 
fins lucrativos, em 1990, por meio de uma iniciativa público-privada. O compromisso nacional foi decisivo para cada etapa da evolução do CIDEIM. Em 2009, o Programa Especial para Pesquisa e Trei-namento em Doenças Tropicais (TDR, na sigla em inglês) selecionou o CIDEIM para ser o centro regional de treinamento nas Américas. Os centros regionais de treinamento colaboram para melhorar a qualidade científica e a competitividade da pesquisa em saúde nos países de baixa e média renda. ${ }^{8,9}$

No CIDEIM, equipes interdisciplinares delineiam e realizam pesquisa básica, translacional, aplicada e de implementação em parceria com autoridades de saúde pública, universidades e comunidades. Os projetos existentes permitem que profissionais em formação realizem pesquisa supervisionada em conjunto com equipes de pesquisadores nacionais e internacionais. A pesquisa prática é complementada por cursos de capacitação, que podem ser presenciais, a distância ou uma combinação dos dois formatos. Os cursos incluem gestão de dados, planejamento de projetos, boas práticas clínicas, planejamento de análise de dados, tecnologia da informação e comunicação na pesquisa em saúde, ética em pesquisa e integridade científica. Essa estratégia promove o cumprimento de padrões internacionais na pesquisa e a participação em agendas de pesquisa nacionais e internacionais. Um aspecto importante dos cursos é sua concepção para replicação mediante formação de instrutores e colaboração com instituições da rede regional para desenvolver e adaptar os módulos de treinamento.
Como centro regional de treinamento, o CIDEIM tem uma estratégia de capacitação voltada para instituições (Quadro 2). A OPAS ajuda a identificar instituições participantes e oferece apoio logístico para a difusão regional. Desde 2009, o CIDEIM treinou mais de 2.000 pesquisadores, transferiu capacidade de treinamento para instituições da rede na América Central, na América do Sul e no Caribe, e realizou treinamento inter-regional em colaboração com centros regionais de treinamento na Indonésia, no Cazaquistão e em Gana. ${ }^{10}$

\section{FORTALECIMENTO INSTITUCIONAL POR COLABORAÇÃO NORTE-SUL E SUL-SUL: ƯNIDADE DE EPIDEMIOLOGIA CLÍNICA DA UNIVERSIDADE DAS ÍNDIAS OCIDENTAIS}

A unidade de pesquisa em epidemiologia da Universidade das Índias Ocidentais (UWI, na sigla em inglês) foi criada em 1999 como parte do Instituto de Pesquisa em Medicina Tropical, atual Instituto para Pesquisa em Saúde do Caribe (CAIHR, na sigla em inglês). A necessidade de treinamento regional em epidemiologia foi reconhecida pela unidade, que criou um programa de mestrado em 2005, seguido por um programa de doutorado.

Em maio de 2007, os participantes da $40^{\text {a }}$ Reunião do Comitê Consultivo de Pesquisa em Saúde da OPAS propuseram a criação de uma equipe de epidemiologistas para aumentar a produção e o uso de evidências para informar políticas de saúde pública no Caribe. ${ }^{11}$
Os representantes da OPAS comprometeram-se a apoiar articulações entre a INCLEN e a Universidade das Índias Ocidentais. A reunião tornou-se o catalisador de uma série de desenvolvimentos, com a unidade de pesquisa em epidemiologia como seu ponto focal.

A unidade de epidemiologia clínica da Universidade das Índias Ocidentais (UEC/UWI), criada em fevereiro de 2009, contava com membros do corpo docente da unidade de epidemiologia e de outros departamentos da universidade. ${ }^{12}$ Os membros frequentaram um curso de treinamento, intitulado "Planejamento e avaliação efetivos de projetos em pesquisa biomédica," oferecido pelo CIDEIM na América Latina e no Caribe. ${ }^{9}$ Um importante elemento de capacitação do curso é o treinamento de participantes locais para ministrá-lo, permitindo que a Universidade das Índias Ocidentais reproduza o treinamento na Jamaica e em outros locais. Em seguida, a unidade ofereceu treinamento em planejamento e avaliação de projetos em pesquisa biomédica para mais de 200 profissionais caribenhos. A metodologia foi incorporada a cursos de pós-graduação na Universidade das Índias Ocidentais e na Universidade de St. George, Granada. ${ }^{13}$ $\mathrm{O}$ pessoal da Universidade das Índias Ocidentais também recebeu treinamento em síntese de conhecimento e tradução da pesquisa em prática, o que facilitou o lançamento da Colaboração Cochrane no Caribe em junho de $2013^{14}$ (Quadro 3).

Essas habilidades e experiências ajudaram a consolidar o programa de epidemiologia da Universidade das Índias Ocidentais. O resultado foram 19 epidemiologistas capazes de lidar com

Quadro 2: Centro Internacional de Entrenamiento e Investigaciones Médicas (CIDEIM)

O treinamento é essencial para a missão e o programa de pesquisa do CIDEIM. A capacidade de pesquisa é desenvolvida por diversas estratégias de treinamento, que aproveitam e complementam programas de pós-graduação em universidades nacionais. As pesquisas em andamento oferecem uma plataforma para pesquisa clínica, laboratorial e de campo com tutoria desde o pré-doutorado até o pós-doutorado. É importante notar que os membros do corpo docente e os estudantes participam de cursos de capacitação para pesquisa e de cursos de pós-graduação eletivos on-line.

Desde 2007, o Programa Global de Treinamento de Pesquisa em Doenças Infecciosas do Centro Internacional Fogarty dos Institutos Nacionais de Saúde dos EUA tem apoiado cursos de pós-graduação on-line com duração de um semestre. Estes abrangeram temas avançados pertinentes à biomedicina e ciências da saúde e foram organizados e realizados pelo CIDEIM em colaboração com a Escola de Saúde Pública da Universidade de Yale. Há palestras e discussões ao vivo em ambiente virtual com especialistas nacionais e internacionais, e os cursos são registrados para crédito como disciplinas eletivas nos programas de pós-graduação das universidades participantes. O material do curso, inclusive palestras gravadas e avaliações, é acessado e gerenciado na plataforma Moodle de aprendizagem aberta. Mais de 1.000 pesquisadores e trainees de 30 universidades colombianas, 12 institutos, instituições do setor de saúde, indústria e 17 universidades/institutos regionais da América Latina e do Caribe participaram desses cursos eletivos. 
Quadro 3: Unidade de epidemiologia clínica da Universidade das Índias Ocidentais (UEC/UWI)

Os estágios para candidatos a doutorado da UEC/UWI na Universidade de Ottawa e na Universidade Johns Hopkins levaram ao surgimento de um interesse especial em revisões sistemáticas e atenção à saúde baseada em evidências.

O resultado foi o primeiro seminário de treinamento na metodologia de revisão sistemática na Jamaica em março de 2012 (apoiado pelo corpo docente da UEC/UWI, o Centro para Saúde Global da Universidade de Ottawa e a OPAS) e o subsequente lançamento da colaboração Cochrane no Caribe em junho de 2013.

A colaboração Cochrane no Caribe, junto com membros da UEC/UWI, sediou oficinas de treinamento Cochrane anuais no Instituto Caribenho para a Pesquisa em Saúde e deu apoio técnico aos territórios de língua inglesa no Caribe. O centro também facilita a articulação entre pesquisadores locais e internacionais e ministra treinamento em revisões sistemáticas e atenção à saúde baseada em evidências para estudantes universitários e de pós-graduação. A colaboração Cochrane no Caribe apoiou a elaboração e a conclusão de quatro revisões sistemáticas iniciadas por pesquisadores locais, colaborou em mais de 20 revisões sistemáticas e forneceu evidências para a formulação de políticas regionais e nacionais - mais recentemente, na política da indústria de alimentos e nas relações de medicamentos.

as necessidades regionais de pesquisa na comunidade acadêmica, a administração e prestação de saúde, bem como a formulação de políticas. ${ }^{15}$ No início, muitos candidatos buscavam o treinamento para melhorar o desempenho em postos existentes ou preencher postos vagos. Agora, há maior ênfase em oferecer oportunidades de desenvolvimento de pesquisa e planos de carreira para incentivar os diplomados a permanecerem no Caribe.

\section{DESAFIOS PARA MANTER A CAPACITAÇÃO}

$\mathrm{O}$ apoio financeiro para manter muitas dessas iniciativas é insuficiente. A perda do apoio da Fundação Rockefeller restringiu consideravelmente as atividades da INCLEN e LatinCLEN. A OPAS contribui para as iniciativas regionais de treinamento do CIDEIM e da UEC/ UWI por meio do apoio a oficinas e intercâmbios de pesquisadores. Entretanto, o treinamento e a capacitação dependem de financiamento institucional estável e oportunidades de carreira que atrairão novas gerações de pesquisadores em saúde. O pessoal-chave na UEC/UWI e praticamente todo o pessoal no CIDEIM dependem de financiamento para os projetos. Isso significa que é necessário um equilíbrio entre a apresentação de resultados de projetos e as atividades da capacitação, o que é um desafio para a sustentabilidade. Além disso, há escassez crítica de pessoal capacitado em alguns campos, entre os quais estão a bioestatística, a ciência de implementação, a gestão de pesquisa e a economia em saúde. Atualmente, a capacitação concentra-se em recursos humanos, mas há também necessidade crucial de investimentos na construção e renovação da infraestrutura institucional.

\section{DISCUSSÃO}

Os estudos de caso apresentados neste texto ilustram as diferentes estratégias para desenvolver capacidade de pesquisa nos países de baixa e média renda. ${ }^{16}$ Estas incluem projetos de pesquisa verticais nos quais pesquisadores de países de alta renda orientam e realizam pesquisas específicas em países de baixa e média renda; centros de excelência que concentram o investimento em algumas instituições; parcerias Norte-Sul centradas em uma pergunta de pesquisa específica e redes e consórcios que articulam várias instituições de pesquisa com habilidades complementares para lidar com as dificuldades existentes. ${ }^{16}$ Cada estudo de caso usou combinações de todas as estratégias ao longo do tempo para capacitação individual e institucional.

Embora os projetos verticais possam alcançar os objetivos do projeto, geralmente não produzem capacidade sustentável nos países de baixa e média renda depois de concluídos. A princípio, a INCLEN concentrou-se na capacitação através de bolsas de estudos individuais. Entretanto, constatou que o fortalecimento das instituições pela criação de unidades de epidemiologia clínica com equipes multidisciplinares era crucial para manter os ganhos da capacitação e reter os talentos. ${ }^{4}$

A experiência do CIDEIM ilustra como as colaborações Norte-Sul podem levar à criação de centros de excelência sólidos. Ao longo de 57 anos, o CIDEIM tornou-se um centro de capacitação em pesquisas inovadoras com influência regional e global. Por meio da parceria com a OPAS e instituições regionais da América Latina e do Caribe, o CIDEIM oferece um modelo de custos compartilhados para treinamento em pesquisa. A incorporação do conteúdo de cursos de curta duração sobre boas práticas em pesquisa aos currículos de programas de pós-graduação na região amplia e mantém a capacitação para pesquisa.

A UEC/UWI ilustra o uso de uma combinação de medidas de capacitação institucional regional. A localização da UEC/UWI no CAIHR, uma instituição cuja principal atividade é a pesquisa, foi decisiva para conceder aos profissionais tempo dedicado à pesquisa. A OPAS, juntamente com parceiros regionais $\mathrm{e}$ internacionais, ao apoiar a UEC/UWI na criação de uma equipe de epidemiologistas regionais no Caribe, possibilitou um impacto maior e mais sustentável. ${ }^{13}$

As limitações atuais de financiamento nacional e internacional para a pesquisa não incentivam o investimento na capacitação local no país, que requer muitos anos de apoio contínuo. As redes de pesquisa da América Latina e do Caribe continuam a recorrer ao financiamento de colaborações Norte-Sul. Uma revisão sistemática recente que examinou o desenvolvimento de capacidade de pesquisa constatou que a maioria das pesquisas sobre colaborações Norte-Sul concentrou-se em problemas nessas relações. Muitas publicações afirmaram que há pouquíssimos benefícios para o parceiro sulista. ${ }^{16}$

Várias organizações em todo o mundo demandam que a capacitação seja um componente essencial do financiamento global de pesquisas a fim de reduzir a "fuga de cérebros" dos países de baixa 
e média renda. ${ }^{17}$ As oportunidades de carreira estável em pesquisa constituem um importante fator determinante da pesquisa sustentável e da capacidade de treinamento em pesquisa. Mecanismos de financiamento para capacitação poderiam alavancar estrategicamente qualidades existentes em instituições da América Latina e do Caribe. Um programa de financiamento de pós-doutorado em países de baixa e média renda poderia começar a lidar com essa etapa crucial de transição no desenvolvimento profissional, sobretudo nessa região onde a maioria dos países conta com programas de capacitação em nível de pós-graduação.

Alguns organismos internacionais instituíram mecanismos de financiamento voltados para equipes de pesquisa em países de baixa e média renda. Entre eles figuram os programas de subvenção dos Centros Internacionais de Pesquisa em Doenças Infecciosas e Medicina Tropical dos Institutos Nacionais de Saúde dos EUA, bem como os programas de treinamento Fogarty. O Wellcome Trust oferece diversos programas internacionais de bolsas de estudos, projetos colaborativos e até mesmo auxílios para aquisição de equipamento multiusuário. ${ }^{18}$

As instituições em países de baixa e média renda, sobretudo na América Latina e no Caribe, precisam melhorar a capacidade de fazer previsões acuradas e coerentes do custo total das pesquisas para organismos de financiamento e obter reembolso suficiente de custos indiretos..$^{20}$ ${ }^{21}$ Alguns organismos de financiamento reconhecem as restrições impostas pelo limitado apoio aos custos operacionais e estão dispostos a negociar custos indiretos maiores para essas instituições; outros, porém, limitam esses recursos a um valor menor que o disponível para países de alta renda. ${ }^{19} \mathrm{~A}$ restrição acentuada dos custos indiretos tem impacto negativo no apoio administrativo e no capital investido em infraestrutura e equipamento. É um obstáculo para a participação de pesquisadores e do corpo docente em atividades de treinamento sem fins lucrativos. As instituições em países de baixa e média renda necessitam desse apoio para obter reconhecimento como pontos focais confiáveis para criação e preservação de mudanças. ${ }^{20}$

As soluções nacionais e regionais para o financiamento de instituições na América Latina e no Caribe incluem investimentos no desenvolvimento de sua própria capacidade pela dedicação de uma parcela do orçamento nacional ou de tributos especiais para o financiamento e a infraestrutura de pesquisa. Há também uma oportunidade de colaboração com cientistas da diáspora científica nacional. ${ }^{17}$ Além da abordagem de problemas de saúde urgentes, a melhoria da avaliação do papel essencial das instituições de pesquisa no desenvolvimento nacional pode motivar os formuladores de políticas a reconhecerem a pesquisa em saúde como investimento em vez de gasto. ${ }^{2}$

\section{CONCLUSÕES}

Apesar das diversas dificuldades na capacitação para pesquisa em países de baixa e média renda, os três estudos de caso apresentados mostram que é possível obter resultados positivos pelo uso coordenado de redes existentes e fundos limitados. Esses estudos destacam ainda a importância da colaboração Norte-Sul e Sul-Sul no aumento da capacidade de pesquisa para melhorar a saúde nas Américas. Os componentes essenciais do seu êxito são apoio a líderes comprometidos, treinamento por aproveitamento de iniciativas regionais de treinamento existentes e criação de boas parcerias regionais e internacionais. A presença de instituições de pesquisa capacitadas na América Latina e no Caribe será crucial para a pesquisa em saúde sustentável e equitativa no futuro.

Agradecimentos. A Ana Amelia Correa pela revisão da tradução.

Contribuição dos autores. Todos os autores lideraram ou orientaram muitas das iniciativas descritas neste papel e participaram da conceituação, redação e revisão do texto, aprovando a versão FINAL do artigo apresentado para publicação, e são responsáveis por todas as partes do trabalho com que contribuíram. MKT-R é o garantidor do artigo.

Conflito de interesses. Lemos e compreendemos a política do BMJ sobre a declaração de interesses e não temos interesses relevantes a declarar.

Procedência do artigo e revisão por pares. Encomendado; revisão por pares externa.

Declaração. As opiniões externadas neste manuscrito são de responsabilidade do(s) autor(es) e não refletem necessariamente os critérios e políticas da RPSP/ PAJPH e/ou da OPAS/OMS.

Série Fortalecimiento da pesquisa em saúde nas Américas. Este artigo faz parte de uma série proposta pela OPAS e encomendada pelo BMJ, o qual foi responsável pela revisão por pares, edição e decisão de publicar o artigo, sem participação da OPAS. As despesas referentes à publicação desta série em Open Access foram financiadas pela OPAS. Para ler os outros artigos da série em inglês, visite www.bmj.com/health-research -americas; acesso à série em espanhol e português em https://www.paho .org/journal/es/numeros-especiales / fortalecimiento-investigacion-para -salud-americas

\section{REFERÊNCIAS}

1. Organização Mundial da Saúde. Redução das desigualdades no período de uma geração: igualdade na saúde através da acção sobre os seus determinantes sociais. Relatório Final da Comissão para os Determinantes Sociais da Saúde. 2008. Disponível em: https://www.who.int /social_determinants/thecommission/final report/en/
2. Organização Pan-Americana da Saúde/ Organização Mundial da Saúde. Política de Pesquisa para a Saúde da OPAS. Documento CD49/10 da $49^{\circ}$ sessão do Conselho Diretor da OPAS. 2009. http://www.paho .org/hq/dmdocuments/2009/CD49-10 -p.pdf?ua $=1$

3. McKee M, Stuckler D, Basu S. Where there is no health research: what can be done to fill the global gaps in health research? PLoS Med 2012;9:e1001209. Disponível em inglês em: https://journals.plos.org / plosmedicine / article?id=10.1371 / journal .pmed.1001209. doi:10.1371/journal. pmed .1001209

4. Halstead SB, Tugwell P, Bennett K. The International Clinical Epidemiology Network (INCLEN): a progress report. J 
Clin Epidemiol 1991;44:579-89. doi:10.1016 /0895-4356(91)90222-U

5. Morrow RHJr, Lansang MA. The role of clinical epidemiology in establishing essential national health research capabilities in developing countries. Infect Dis Clin North Am 1991;5:235-46.

6. Gómez Restrepo C, Muñoz N S, Ruiz AJ, Lanas F. Latin American Clinical Epidemiology Network Series - Paper 1: The Latin American Clinical Epidemiology Network "LatinCLEN". J Clin Epidemiol 2017;86:71-4. doi:10.1016/j.jclinepi.2016.10 .002

7. Board on Science and Technology for International Development, Institute of Medicine National Academy of Sciences. The US capacity to address tropical infectious disease problems. National Academy Press, 1987. Disponível em inglês em: https://books.google .co.uk/books?id=CpYrAAAAYAAJ\&pg $=$ PR2\&lpg=PR2\&dq=The+US+Capacity +to+Address+Tropical+Infe \%20ctious+ Disease+Problems.+National+Academy +of+Science+1987. +Report+No.\&sou rce $=$ bl\&ots $=$ HsxQz52EoX\&sig $=$ Cb5u 1 -65MEE4PTSqcpTo_\%20d4Y0MY\&hl=en $\& \mathrm{sa}=$ X\&redir_esc $=\mathrm{y} \# \mathrm{v}=$ onepage $\& \mathrm{q}=\mathrm{The}$ $\% 20$ US $\% 20$ Capacity $\% 20$ to $\% 20$ Address $\% 20$ Tropical\%20Infe \%20ctious\%20Disease $\% 20$ Problems. $\% 20$ National\%20Academy $\% 20$ of $\% 20$ Science $\% 201987 . \% 20$ Report $\% 20$ No.\&f=false

8. World Health Organization. Regional training centers. An introduction to the TDR network. 2015. Disponível em inglês em: https:/ / www.who.int/tdr/news / 2015 /regional_training_centers/en/

9. Ogundahunsi OA, Vahedi M, Kamau EM, et al. Strengthening research capacityTDR's evolving experience in low- and middle-income countries. PLoS Negl Trop Dis 2015;9:e3380. doi:10.1371/journal .pntd.0003380. Disponível em inglês em: https://journals.plos.org/plosntds /article?id=10.1371/journal.pntd.0003380.

10. Alger J, Gómez L, Jaramillo A, Saravia NG, Cuervo LG. Reunión de la Red Inter-Regional de Planeación y Evaluación Efectivas de Proyectos de Investigación para la Salud, Cali, Colômbia, Abril 2010. Rev Med Hondur 2010;78:97-100. Disponível em espanhol em: https://www.paho .org/hq/dmdocuments / 2012/44-CAIS -2010-Formacion-Gestion-Investigacion -Alianza-CIDEIM-OPS-TDR.pdf

11. Comité Asesor de Investigaciones en Salud (CAIS). Organización Panamericana de la Salud/ Organización Mundial de la Salud (OPS/OMS). $40^{\circ}$ CAIS: Panorama de la situación de las políticas de investigación en las Américas y propuesta para el desarrollo de una política en investigación en salud de la Organización. 2007. Disponível em espanhol em: http://www1.paho.org /spanish/DD/IKM/RC/CAIS-2007-40-05 -Panorama.pdf

12. University of the West Indies. UWI admitted to International Epidemiology Network. Press release. Disponível em inglês em: https://www.mona.uwi.edu /marcom/uwinotebook/entry/3492

13. Pan American Health Organization/World Health Organization. Report on strengthening research capacities for health in the Caribbean, 2007-2017. Washington, DC: OPAS/OMS, 2017. Disponível em inglês em: http://iris.paho.org/xmlui/handle /123456789/34342

14. Cochrane Caribbean. Cochrane Caribbean launch. Press release, 2013. Disponível em inglês em: https://caribbean.cochrane.org /cochrane-caribbean-launch
15. Tulloch-Reid MK. The University of the West Indies Clinical Epidemiology Unit: collaborating to improve health. J Clin Epidemiol 2015;68:1099-100. doi:10.1016 /j.jclinepi.2015.04.012

16. Franzen SR, Chandler C, Lang T. Health research capacity development in low- and middle-income countries: reality or rhetoric? A systematic meta-narrative review of the qualitative literature. BMJ Open 2017;7:e012332. doi:10.1136/bmjopen-2016 $-012332$.

17. Saravia NG, Miranda JF. Plumbing the brain drain. Bull World Health Organ 2004;82:608-15.

18. Wellcome Trust. Scheme finder. United Kingdom: Research Council UK, 2018. Disponível em inglês em: https://wellcome .ac.uk/funding/scheme-finder

19. United States National Institutes of Health NIH grants policy statement. 2017. Disponível em inglês em: https://grants.nih .gov/grants/policy/nihgps/html5/section _7/7.4_reimbursement_of_facilities_and administrative_costs.htm

20. Lucas S. How to fund think tanks effectively even when you can't. William and Flora Hewlett Foundation, 2017. Disponível em inglês em: https://hewlett.org /fund-think-tanks-flexibly-even-cant/

21. Council on Health Research and Development. Fair research contracting. Genebra, Suíça, 2018. Disponível em inglês em: http://www.cohred.org/frc/

Como citar (artigo original): BMJ 2018;362:k2456. http:/ /dx.doi.org/10.1136/bmj.k2456

Manuscrito original em inglês publicado em 16 de julho de 2018 